\title{
EXPÉRIMENTATION ET ANALYSE THERMIQUE D'UN CONCENTRATEUR HÉMISPHÉRIQUE STATIONNAIRE SOUS LES CONDITIONS CLIMATIQUES À OUGADOUGOU, BURKINA FASO
}

\author{
Ouédraogo Salifou, ", Ky Thierry S. M., Konfé Amadou, Kam Sié, Bathiébo D. Joseph \\ Laboratoire d'énergies thermiques renouvelables, Université Joseph KI-ZERBO, Ouagadougou, Burkina Faso \\ *osalif12@gmail.com
}

\section{INFOS SUR L’A R T I C L E}

Historique de l'article:

: 09 février 202

Accepté le : 18 février 2021

Mots-Clés: Concentrateur hémisphérique, stationnaire, énergie solaire, température intermédiaire

Keywords : Hemispherical concentrator, stationary, solar energy, intermediate température

\begin{abstract}
R É S U M É
Les concentrateurs solaires constituent une technologie adaptée à l'exploitation de l'énergie solaire à haute température mais aussi à des températures intermédiaires. Cette technologie est utilisée dans le séchage, le chauffage d'eau sanitaire ou dans la production d'électricité. Cependant elle utilise un système de traque solaire qui est trop complexe et couteuse pour les pays moins équipés et ayant un fort potentiel solaire. Dans cette étude, on s'intéresse à l'expérimentation et à l'analyse thermique d'un concentrateur hémisphérique stationnaire. La résolution numérique des équations de la caustique d'un concentrateur sphérique a permis de déterminer les dimensions et la position du receveur, nécessaires à la conception du modèle physique et au montage du dispositif expérimental. Les résultats de la simulation numérique à $3 \mathrm{D}$ avec le logiciel Comsol5.3a ont permis de mettre en évidence le tracé des rayons et le profil du flux concentré sur le receveur. Les résultats obtenus expérimentalement montrent que le receveur et l'air à l'intérieur ont atteint respectivement une température maximale de $224{ }^{\circ} \mathrm{C}$ et $97,6{ }^{\circ} \mathrm{C}$. Le dispositif de concentration solaire étudié est donc techniquement favorable à des applications thermiques nécessitant des températures intermédiaires.
\end{abstract}

\begin{abstract}
A B S T R A C T
This technology is used in drying, domestic water heating or in the production of electricity. However, it uses a solar tracking system that is too complex and expensive for countries with less equipment and high solar potential. In this study, we are interested in the experimentation and thermal analysis of a stationary hemispheric concentrator. The numerical resolution of the caustic equations of a spherical concentrator allowed to determine the dimensions and the position of the receiver, necessary for the design of the physical model and the assembly of the experimental device. The results of the $3 \mathrm{D}$ numerical simulation with the Comsol5.3a software allowed to highlight the ray tracing and the profile of the flow concentrated on the receiver. The results obtained experimentally show that the receiver and the air inside reached a maximum temperature of $224^{\circ} \mathrm{C}$ and $97.6^{\circ} \mathrm{C}$ respectively. The solar concentration device studied is therefore technically favorable for thermal applications requiring intermediate temperatures.
\end{abstract}

\section{INTRODUCTION}

Actuellement utilisés comme une technologie efficace à l'exploitation du rayonnement solaire, les concentrateurs solaires ont fait l'objet d'étude de plusieurs revues dans la littérature [1-6]. De ces différentes études, la configuration géométrie du système et les propriétés optiques des matériaux sont les principaux facteurs qui influent sur les performances optiques et thermiques des concentrateurs solaires. L'influence de ces paramètres est difficile à estimer à priori [7].
La technologie solaire concentrée a la capacité de produire de l'énergie verte distribuable sous forme de chaleur et d'électricité [8]. En outre elle permet de réduire les surfaces d'utilisation, les pertes thermiques et d'obtenir des températures élevées, ce qui constitue un avantage majeur par rapport aux autres technologies d'exploitation de l'énergie solaire non concentrées.

Un concentrateur solaire se définit comme un dispositif permettant de concentrer les rayons solaires sur une cible dans le but de récupérer l'énergie maximale. 
L'objectif principal de la concentration se définit en fonction de l'application [9] :

- $\quad$ pour le photovoltaïque c'est d'obtenir le maximum de luminosité au niveau de la cible,

- pour le solaire thermique c'est d'obtenir une température plus élevée.

Les concentrateurs solaires sont classés selon que la concentration soit ponctuelle, linéaire ou plane, une classification qui nous renseigne sur la forme du receveur à concevoir.

Le concept de SRTA (réflecteur sphérique fixe / absorbeur suiveur solaire) a été créé en 2028 par Berland [10] . Selon ce concept, un concentrateur sphérique concentre toujours le rayonnement solaire le long d'une ligne passant par le centre de la sphère. Cet état de fait est illustré par la figure 1.

Une étude menée par Villasante et al. a montré l'applicabilité du concept pour la collecte de la chaleur solaire à des températures intermédiaires $\left(<150^{\circ}\right)$ [11].

Dans ce travail au dimensionnement du receveur et à l'analyse thermique d'un mini concentrateur sphérique de type stationnaire.
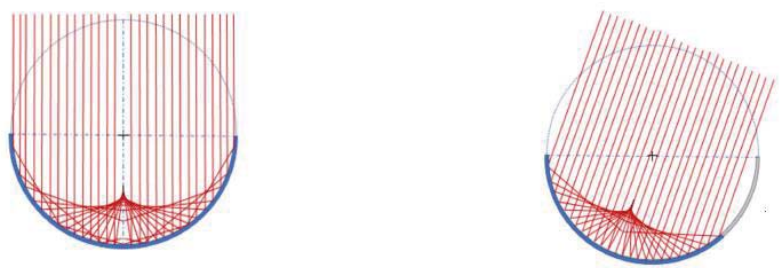

Figure 1: Formation de la tache solaire selon le concept SRTA [11].

\section{MATÉRIEL ET MÉTHODES}

\subsection{Dimensionnement du receveur}

En effet dans 1'approximation de Gauss, lorsqu'un faisceau lumineux arrive parallèlement à l'axe du concentrateur hémisphérique, tous les rayons réfléchis convergent au foyer (F) du concentrateur pour des demiangles d'ouverture de faibles valeurs $\left(<11^{\circ}\right)$, cette approximation est valable pour les concentrateurs sphériques [9]. Toutefois, les $\operatorname{surfaces}(\mathrm{S})$ des concentrateurs sphériques sont en général astigmatiques. Ainsi les rayons réfléchis sont tangents à une surface de révolution appelée, caustique d'aberration sphérique, représentée à la figure 2. Dans cette zone d'aberration sphérique, les rayons réfléchis forment une tache lumineuse dont l'éclairement n'est pas uniforme, appelé disque de moindre diffusion. La position et le rayon du disque de moindre diffusion définiront la position et les dimensions du receveur.

L'équation paramétrique de la caustique encore appelée équation de Néphroïde [12] est :

$\left\{\begin{array}{l}X=R \sin ^{3} \varphi \\ Y=\frac{R}{2} \cos \varphi\left(1+2 \sin ^{2} \varphi\right)\end{array}\right.$
$X \cos (2 \varphi)+Y \sin (2 \varphi)-R \sin \varphi=0$

Où $\mathrm{X}$ et $\mathrm{Y}$ sont les coordonnées du pont d'intersection(T) de la caustique et de la droite (AM). $X$ désigne le rayon du disque de moindre diffusion et $\mathrm{Y}$ sa position dans le concentrateur.

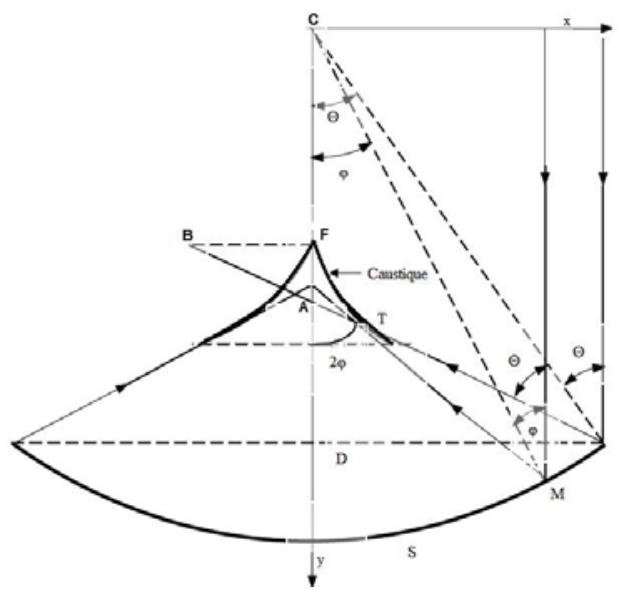

Figure 2: Caustique d'un concentrateur sphérique[12]

$\Theta, \varphi$ désignent respectivement les angles d'ouverture au centre du concentrateur et au foyer.

La résolution de l'équation (2) pour une valeur de $\theta=30^{\circ}$, conduit à : $\varphi=17,37^{\circ}$, d'où :

$$
\begin{aligned}
& X=26,47 \cdot 10^{-3} R \\
& Y=0,562 R
\end{aligned}
$$

En prenant en compte du diamètre apparent du soleil, le rayon $\mathrm{Ra}$ du disque aura pour expression [6] :

$$
R_{a}=(R \sin \varphi+X) \times \frac{\cot (2 \varphi)}{\cot (2 \varphi+0,266)}-R \sin \varphi
$$

Ky et al. [13] ont montré que suivant la course apparente du soleil, le disque de moindre diffusion décrit un demicercle de rayon Y à l'intérieur d'un concentrateur hémisphérique, illustrée à la figure 3 . La valeur du rayon du demi-cercle est voisine de celle de la moitié de la valeur du rayon du concentrateur.

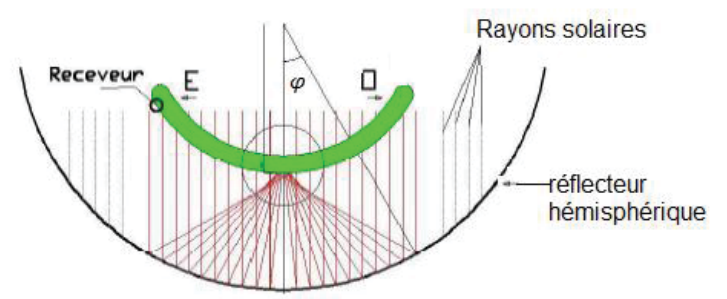

Figure 3: Modélisation du principe

A la lumière de ce travail, ils ont conçu un système muni d'un receveur cavité rotatif, en forme de cerceau, avec un système de traque quasi-fixe. Les résultats d'une étude expérimentale du dispositif ont montré sa rentabilité technique et économique. Une température maximale de $300{ }^{\circ} \mathrm{C}$ en fonctionnement, a également été enregistrée [14].

L'équation de la droite (AM) s'écrit : 
Le concentrateur hémisphérique dont nous disposons à un rayon de $0,14 \mathrm{~m}$. Les équation (4) et (5) conduisent donc à : $R a=4,5 \cdot 10^{-3} \mathrm{~m}$ et $Y=8 \cdot 10^{-2} \mathrm{~m}$.

La concentration géométrique $(\mathrm{Cg})$ est définie par l'équation (6) :

$$
C_{g}=\left(\frac{R \sin \varphi}{R_{a}}\right)^{2}
$$

Avec les valeurs obtenues précédemment, on obtient une concentration moyenne de $C_{g} \cong 242$.

\subsection{Description du dispositif conçu}

Un saladier «BLANDA BLANK » d'IKEA est utilisé comme réflecteur hémisphérique. Il est en acier inoxydable, avec une surface intérieure chromée réfléchissante de diamètre $28 \mathrm{~cm}$ et de profondeur $14 \mathrm{~cm}$. Le receveur cavité en cuivre de $0,3 \mathrm{~mm}$ est suspendu à une vitre en verre sodo-calcique transparente de $5 \mathrm{~mm}$ couvrant la surface d'ouverture. La photo du dispositif est représentée à la figure 4 .

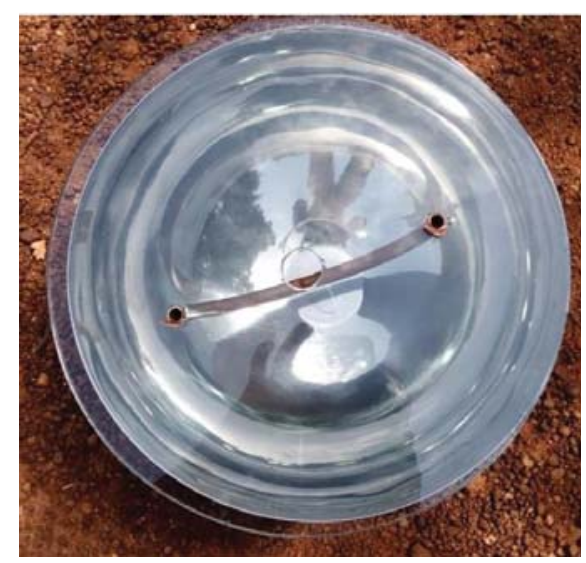

Figure 4: Photo du modèle réalisé

\subsection{Protocole expérimental}

Nous avons réalisé une série de mesures, durant les journées du 29 Octobre et du 11 Novembre 2020, sur le dispositif exposé sur le site de l'Atelier Central de Maintenance $\left(12,21^{\circ}\right.$ Nord et $-1,25^{\circ}$ Ouest) de 1'Université Joseph Ki-Zerbo de Ouagadougou, BurkinaFaso.

Ces mesures ont concerné :

- la température du receveur, de l'air à l'intérieur de l'hémisphère, de l'air ambiant en utilisant des thermocouples de type $\mathrm{K}$ à gaine blindée ;

- le rayonnement global à l'aide d'un pyranomètre SR03-05 de la marque Hukseflux, car ne disposant pas d'un pyrhéliomètre pour mesurer l'éclairement solaire direct.

Les thermocouples et le pyranomètre sont connectés à un enregistreur automatique de données, Midi LOGGER GL200A de la marque GRAPHTEC, programmé à une cadence de $2 \mathrm{mn}$. La figure 4 illustre le montage expérimental.

Les caractéristiques techniques des instruments utilisés sont mentionnées dans le tableau 1.
Tableau 1 : Caractéristiques techniques des instruments de mesure

\begin{tabular}{lcc}
\hline Instrument & Plage de mesure & Exactitude \\
\hline $\begin{array}{l}\text { Thermocouple } \\
\text { type K }\end{array}$ & -40 à $1000{ }^{\circ} \mathrm{C}$ & $\mp 1,5 \%$ \\
$\begin{array}{l}\text { Pyranomètre } \\
\text { SR03-05 }\end{array}$ & 0 à $1600 \mathrm{~W} \cdot \mathrm{m}^{-2}$ & $\mp 1,8 \%$ \\
\hline
\end{tabular}

La surface d'ouverture du concentrateur est disposée horizontalement et l'axe du receveur orienté Est-Ouest et ce pour toute la journée.

La vitesse du vent est faible et son influence et les pertes par convection sont négligeables.

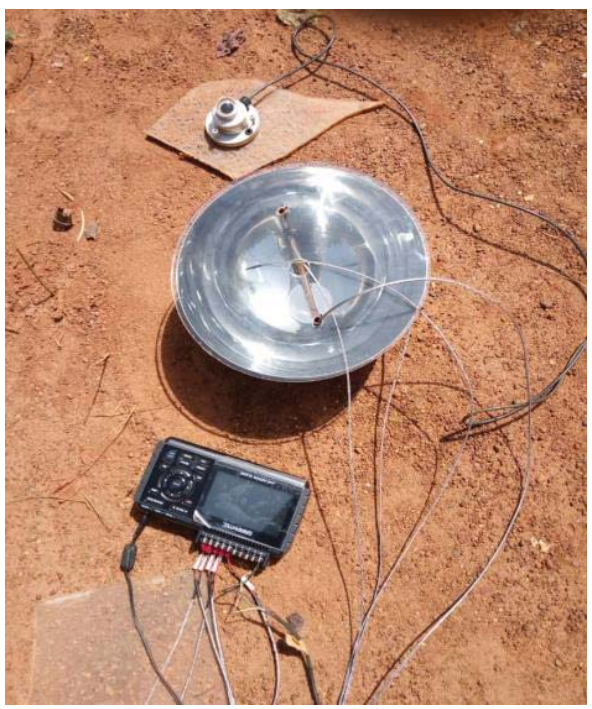

Figure 5: Photo du montage expérimental

\section{RÉSULTATS ET DISCUSSION}

\subsection{De l'expérience}

La figure 6 présente l'évolution temporelle rayonnement global des journées de mesure.
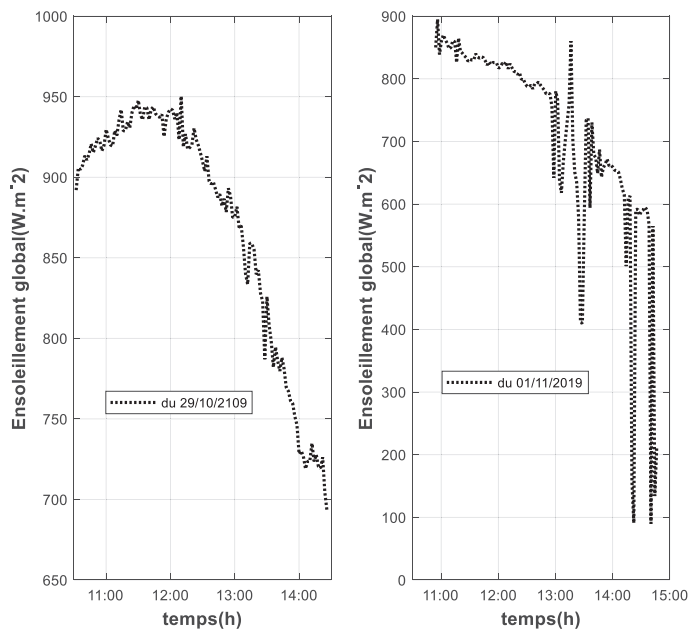

Figure 6: Évolution temporelle du rayonnement solaire global

Les courbes présentent des fluctuations importantes dans l'intervalle de temps de mesure et des valeurs maximales de 947,8 W.m-2 et 895 W.m-2 ${ }^{-2}$, respectivement aux dates du 29 Octobre et du 11 Novembre 2020. Ces fluctuations, 
résultant d'une nébulosité, sont plus importantes au cours de la deuxième journée.

La figure 7 illustre l'évolution de la température aux points de mesure indiqués précédemment au cours des deux journées.
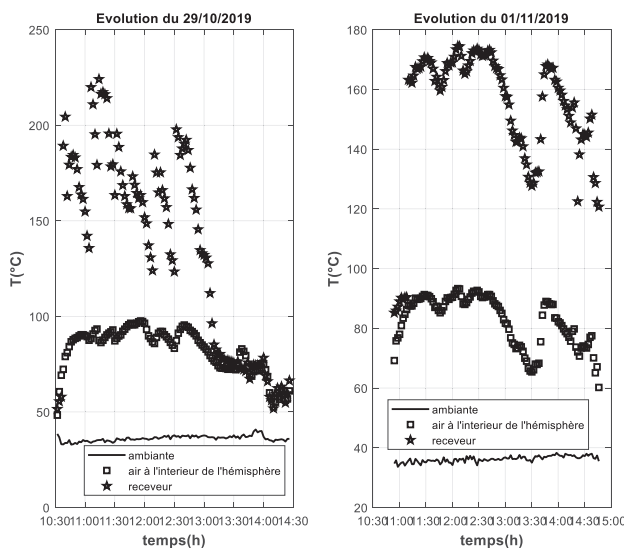

Figure 7 : Évolution des températures de l'air ambiant, $d u$ receveur et de l'air à l'intérieur de l'hémisphère

L'évolution des températures du receveur et de l'air interne présentent également des fluctuations et atteignent respectivement les valeurs maximales de 224,1 ${ }^{\circ} \mathrm{C}$ et $97,6{ }^{\circ} \mathrm{C}$ au cours de la journée du 29 Octobre; $174,5^{\circ} \mathrm{C}$ et $93,3{ }^{\circ} \mathrm{C}$ au cours de la journée du 01 Novembre. Ces températures sont plus importantes au niveau du receveur et ce d'autant plus que le rayonnement est élevé.

\subsection{De la simulation}

Le tracé des rayons à trois (03) dimensions a été obtenu en considérant les hypothèses suivantes :

- les rayons incidents ont une intensité de 947,8 W.m

2 ,

- la surface du réflecteur est réelle (non idéale),

- le receveur est en cuivre,

- le régime est stationnaire.

Les figures 8 a) et b) présentent respectivement le tracé de la marche des rayons lumineux et du profil du flux concentré sur la surface du disque de moindre diffusion.
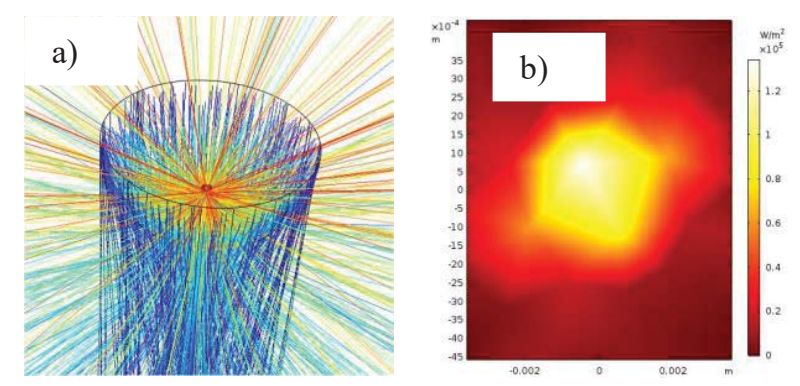

Figure 8 : a) Tracé des rayons réfléchis su plan focal, b) Profil du flux concentré

La figure 8 a) montre que les rayons réfléchis sont projetés sur le disque de moindre diffusion. Cette projection permet de caler la position et les dimensions précédemment déterminées du receveur.
La projection des rayons réfléchis forme le flux concentré illustré à la figure $8 \mathrm{~b}$ ). Cette figure montre un flux non uniforme sur la surface totale du disque. Un flux dont l'intensité est plus importante au centre du disque et varie entre $2.10^{4}-12.10^{4} \mathrm{~W} \cdot \mathrm{m}^{-2}$.

Dans les mêmes conditions on a obtenu le profil de températures du receveur présenté à la figure 9 .

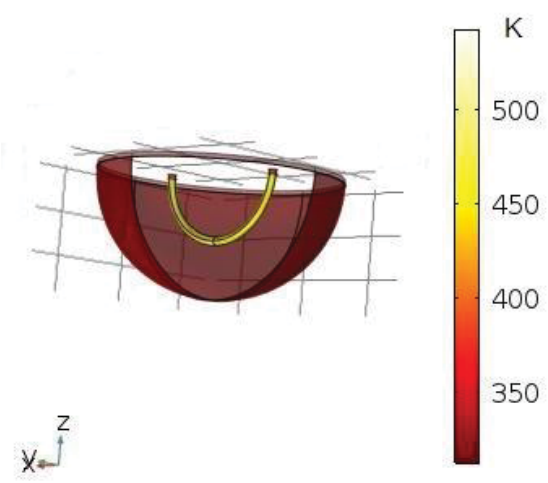

Figure 9 : Distribution de la température du receveur

Ce profil montre que le receveur atteint une température maximale de $500 \mathrm{~K}\left(227^{\circ} \mathrm{C}\right)$. Soit un écart de $3^{\circ} \mathrm{C}$ avec celle obtenue expérimentalement.

\section{CONCLUSION}

Dans ce travail, l'accent est mis sur l'analyse du comportement thermique du receveur. Des températures supérieures à $200{ }^{\circ} \mathrm{C}$ sont atteintes expérimentalement par le receveur, malgré des journées moins ensoleillées, ce qui met en exergue la capacité du dispositif d'atteindre les températures intermédiaires.

Le dépoussiérage est la seule maintenance appliquée à notre dispositif au cours de son expérimentation, ce qui ne nécessite donc pas un coût important. Les résultats de la simulation avec le logiciel Comsol 5.3a, tels que le tracé des rayons et le profil du flux concentré, ont permis d'observer la formation de la tache lumineuse sur le receveur, confirmant ainsi le bon dimensionnement théorique du receveur. Il a été montré également que dans les conditions de l'expérience du 29 Octobre, le receveur atteint une température maximale de $227^{\circ} \mathrm{C}$, soit un écart moyen de températures de $3{ }^{\circ} \mathrm{C}$ ou $1,4 \%$ avec celle obtenue expérimentalement, par ailleurs inférieur à la tolérance du thermocouple qui est de 1,5\%. Des similitudes existent donc entre les résultats expérimentaux et ceux de la simulation, permettant de valider notre modèle physique.

$\mathrm{Au}$ vue des températures atteintes, ce dispositif peut être utilisé pour le chauffage d'eau sanitaire ou dans le collecteur d'un capteur plan solaire.

\section{REMERCIEMENTS}

Nous remercions l'ISP de l'Université d'Uppsala, en Suède, pour son soutien au projet BUF01.

\section{Références}

[1] T. Arvind and T. Shyam, "Handbook of Solar Energy/ Theory, Analysis and Applications," in Energy Systems in Electrical Engineering, Media 
Singapore, 2016, p. 774.

[2] B. M. Pakouzou, M. S. T. Ky, S. T. Gbembongo, and G. P. Ouedraogo, "Thermal Performance of a Receiver Located in the Caustic Area of a Cylindro-Parabolic Solar Concentrator," Phys. Sci. Int. J., vol. 16, no. 3, pp. 1-14, 2017.

[3] D. Guerraiche, B. Ammar, and H. Ben Moussa, "Facteurs optiques et géométriques caractérisant un concentrateur cylindro-parabolique," Rev. des Energies Renouvelables, vol. 14, no. 02, pp. 229 238, 2011.

[4] V. S. Reddy, S. C. Kaushik, and S. K. Tyagi, "Exergetic analysis and performance evaluation of parabolic trough concentrating solar thermal power plant ( PTCSTPP )," Energy, vol. 39, no. 1, pp. 258-273, 2012.

[5] Y. Boukhchana, A. Fellah, and A. Ben Brahim, "Etudes théorique et expérimentale des performances d ' un capteur solaire cylindroparabolique," in Congrès International sur les Energies Renouvelables et l'Environnement, 2010, pp. 1-9.

[6] S. M. T. Ky, M. Pakouzou, B. Dianda, M. Ousmane, S. Kam, and D. J. Bathiebo, "Air Heating In A Steady Hemispherical Concentrating System For Various Applications, " Int. J. Curr. Res., vol. 10, no. 02, pp. 65449-65454, 2018.

[7] A. Arconada and F. Papini, "Bilan thermique des convertisseurs de l'énergie solaire : rôle de la sélectivité et de la concentration," Rev. Phys. Appliquée, vol. 15, no. 2, pp. 163-167, 2080.
[8] S. Cohen and G. Grossman, "Development of a solar collector with a stationary spherical reflector / tracking absorber for industrial process heat," Sol. ENERGY, 2015.

[9] T. S. M. KY, “Analyse Des Images Optiques Des Concentrateurs Solaires. Application Pour La Conception De Fours A Pain," Ouaga I Pr Joseph KI-ZEBO, Ouagadou, Burkina-Faso, 2016.

[10] W. G. Steward and F. Kreith, "Stationary concentrating reflector cum tracking absorber solar energy collector : optical design characteristics," Appl. Opt., vol. 14, no. 7, pp. 1509-1512, 2075.

[11] C. Villasante et al., "Low-Cost Solar Electricity Using Stationary Solar Fields; Technology Potential and Practical," energies, vol. 13, no. 1816, pp. 1-14, 2020.

[12] M. KHALED, "Conception et réalisation d'un concentrateur sphérique," Universite Mentouri Constantine, CONSTANTINE, ALGERIE, 2008.

[13] S. M. T. Ky, B. Dianda, E. Ouedraogo, S. Ouedraogo, and D. J. Bathiebo, "Novel Natural Convection Process : Indirect Solar Dryer Built with Spherical Novel Application to Tomato Drying .," Elixir Therm. Eng., vol. 122, pp. 5161551620, 2018.

[14] T. S. M. Ky, B. Dianda, M. Ousmane, M. Pakouzou, and D. J. Bathiebo, "Optical and Thermal Performance Analysis of a Steady Spherical Collector with a Crescent- shaped Rotating Absorber," vol. 6495, no. 4, pp. 234-245, 2017. 


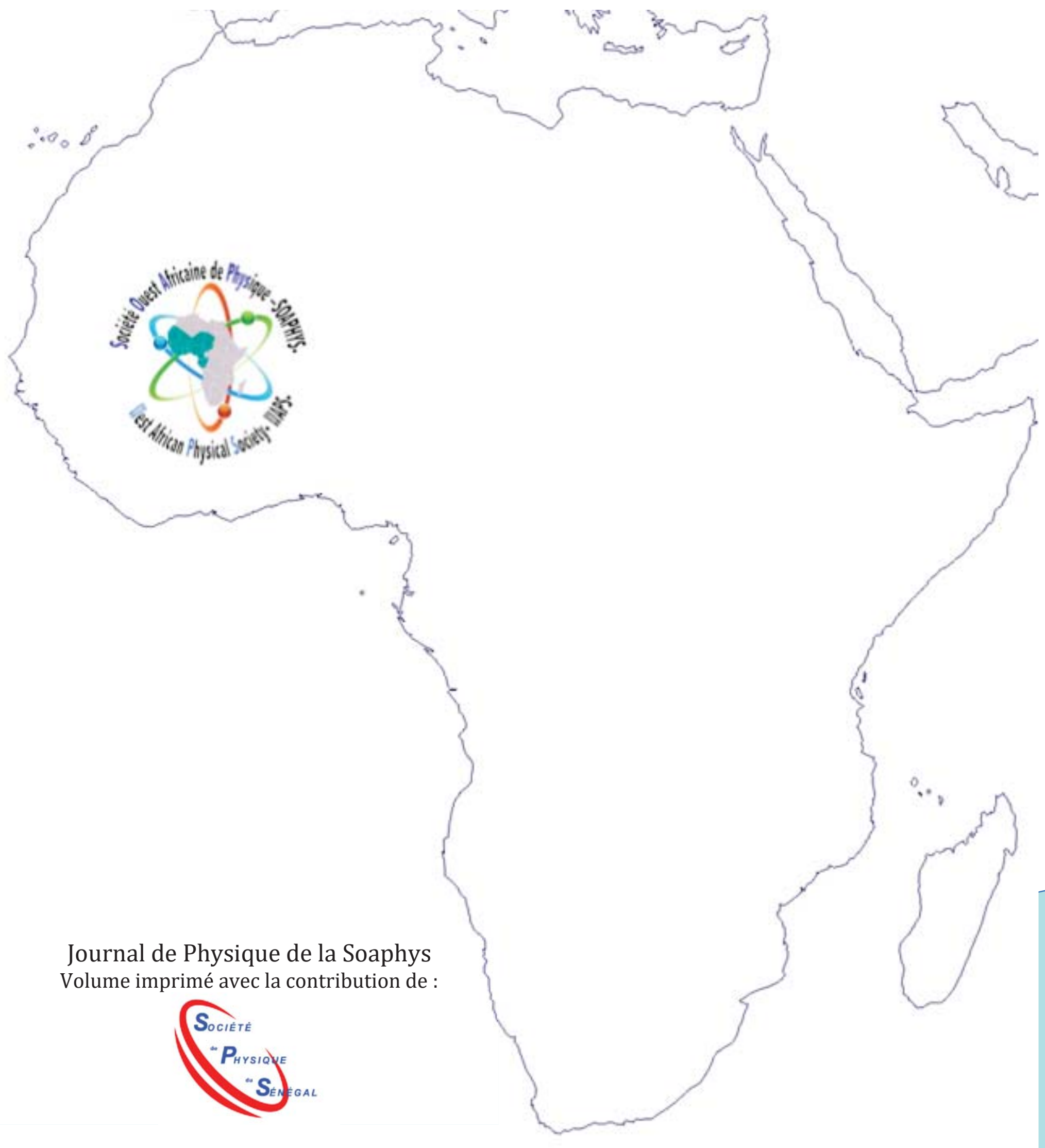

